\title{
Comparison of starch films and effect of different rice starch-based coating formulations on physical properties of walnut during storage time at accelerated temperature
}

\begin{abstract}
Effects of different rice starch-based coating formulations on physical properties of walnut were studied during 20 days storage at accelerated temperature. Results from different starch films indicated there were nonsignificant differences in water and oxygen barrier properties of the starch films. However, significant (pட்105) differences were present in the elongation at break characteristics between the corn and rice films. Tensile strength varied significantly between edible films made from rice starch with that of wheat and corn. Melting temperature (Tm) of wheat and corn starch films was higher than rice starch films (152.77 PRC). Thus, rice starch in combination with different additives such as chitosan, emulsifier, and red palm oil, which improve the properties of rice starches have been chosen to coat dried walnut kernels. The coated walnuts were significantly (p亩105) lighter than the control group. Results on different functional ingredients also showed significant variations, for example; yellowness of walnut in the presence of chitosan was lower among the coating formulas (p Ĺ⿱㇒冋)5). However, the variations in textural characteristics of walnuts with different coating formulas

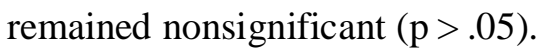

Keyword: Walnut; Coating; Starch films; Rice starch 